\title{
A new approach to quantized stabilization of a stochastic system with multiplicative noise
}

\section{Li Wei and Yuanhua Yang}

\section{"Correspondence:}

weili@mail.sdu.edu.cn

School of Control Science and

Engineering, Shandong University,

Jinan, China

\begin{abstract}
A new quantization-dependent Lyapunov function is proposed to analyze the quantized feedback stabilization problem of systems with multiplicative noise. For convenience of the proof, only a single-input case is considered (which can be generalized to a multi-input channel). Conditions for the systems to be quantized mean-square poly-quadratically stabilized are derived, and the analysis of $H_{\infty}$ performance and controller design is conducted for a given logarithmic quantizer. The most significant feature is the utilization of a quantization-dependent Lyapunov function, leading to less conservative results, which is shown both theoretically and through numerical examples.
\end{abstract}

Keywords: multiplicative noise; discrete-time systems; mean-square stability; logarithmic quantizer; Lyapunov function

\section{Introduction}

Rapid advancement of digital networks has witnessed a growing interest in investigating efforts of signal quantization on feedback control systems. The emerging networkbased control system where information exchange between the controller and the plant is through a digital channel with limited capacities has further strengthened the importance of the study on quantized feedback control. Different from the classical control theory where data transmission is assumed to have an infinite precision, transmission subject to quantization or limited data capacity in digital networks, the tools in classical control theory may be invalid, so new tools need to be developed for the analysis and design of quantized feedback systems.

The study of quantized feedback control can be traced back to [1]. Most of the early research focuses on the understanding and mitigation of the quantization effects, while the quantization error is considered to impair the performance [2]. In modern control theory where the quantizer is always considered as an information encoder and decoder, one main problem is how much information has to be transmitted in order to make the system achieve a certain objective for the closed-loop system. For a discrete-time system with a single-input channel, when the static quantizer is considered, [3] shows the minimum data rate for the system to be stabilized is proved to be characterized by the unstable roots of the system matrix, and the coarsest quantizer is logarithmic. [4] considers the case when the input channel subject to Bernoulli packets dropouts, the minimum data rate is related not only to the unstable roots of the system matrix, but also with the packets dropout probability. As for a discrete-time system with single input subject to multiplicative noises

(c) 2013 Wei and Yang; licensee Springer. This is an Open Access article distributed under the terms of the Creative Commons Attribution License (http://creativecommons.org/licenses/by/2.0), which permits unrestricted use, distribution, and reproduction in any medium, provided the original work is properly cited. 
in [5], the coarsest static quantizer for the system to be quadratically mean-square stabilized is proved to be logarithmic with infinite levels, and the quantization density can be approximated by solving a Riccati equation; comprehensive study on feedback control systems with logarithmic quantizers is not given. A sector bound approach is proposed in [6] to characterize the quantization error caused by a logarithmic quantizer, by which many quantized problem can be solved by the robust tools. The results are also extended to adaptive control in $[7,8]$ and the LQR-type problem in [6]. Based on the characterization of the quantized error, [9] gives less conservative conditions of the quantization density to achieve stability by studying the properties of the logarithmic quantizer further; [10] use a method based on Tsypkin-type Lyapunov functions to study the absolute stability analysis of quantized feedback control of a discrete-time linear system, less conservative conditions than those in the quadratic framework are derived. [11] showed that a finite-level logarithmic quantizer suffices to approach the well-known minimum average data rate for stabilizing an unstable linear discrete-time system under two basic network configurations, and explicit finite-level logarithmic quantizers and the corresponding controllers to approach the minimum average data rate are derived. For networked systems, [12] gives the quantized output-feedback controller for the control with data packets dropout.

In this paper, a new approach to the analysis and synthesis of quantized feedback control for stochastic systems with multiplicative noise is proposed. Using logarithmic quantized state-feedback control, results for mean-square stabilization and $H_{\infty}$ performance analysis as well as the controller synthesis are given. Less conservative results are derived by the utilization of a quantization-dependent Lyapunov function, which is shown both theoretically and through a numerical example.

Notations: $P>0(P \geq 0)$ means $P$ is a symmetric positive (semi-positive) matrix. $P^{T}$ stands for the transposition of matrix $P$. The space of a square summable infinite sequence is denoted by $l_{2}[0, \infty)$, and for $w=\{w(t)\} \in l_{2}[0, \infty)$, its norm is given by $\|w\|_{2}=$ $\sqrt{\sum_{0}^{\infty}|w(t)|^{2}}$.

\section{Stability and stabilization}

\subsection{Problem formulation}

Consider the following linear discrete-time systems with multiplicative noise:

$$
x(t+1)=\left(A+A_{0} \xi(t)\right) x(t)+\left(B+B_{0} \xi(t)\right) u(t), \quad x(0)=x_{0},
$$

where $x(t) \in \mathcal{R}^{n}$ is the system state vector with known initial state $x_{0} ; u(t) \in \mathcal{R}^{m}$ is the control input; $\xi(t) \in \mathcal{R}$ is the process noise with $E \xi(t)=0, E \xi(t) \xi(j)=\sigma^{2} \delta_{t j}$, and is uncorrelated with initial state $x_{0}$. As proved in [5], the coarsest static quantizer for the system (1) to be quadratically mean-square stabilized via quantized state-feedback is proved to be logarithmic. Suppose $u$ is a scalar that has to be quantized, the logarithmic quantizer is in the following form:

$$
q(u)= \begin{cases}u_{i} & \text { if } \frac{1}{1+\delta} u_{i}<u \leq \frac{1}{1-\delta} u_{i}, u>0 \\ 0 & \text { if } u=0 \\ -Q(-u) & \text { if } u<0\end{cases}
$$


with quantization levels as

$$
U=\left\{ \pm u_{i}: u_{i}=\rho_{i} u_{0}, i=1,2, \ldots\right\} \cup\left\{ \pm u_{0}\right\} \cup\{0\}, \quad 0<\rho<1, u_{0}>0,
$$

where $\rho$ is the quantized density of the logarithmic quantizer $q$, which can be computed using the approach given in [5], with

$$
\delta=\frac{1-\rho}{1+\rho}
$$

For the multi-input case with different quantizers, the state-feedback control without quantization is in the form of

$$
v(t)=\left[\begin{array}{llll}
K_{1} x(t) & K_{2} x(t) & \cdots & K_{m} x(t)
\end{array}\right],
$$

which has to be transmitted through a digital network subject to logarithmic quantizers as given in (2), and denote the quantized control as

$$
u(t)=q(v(t))=\left[\begin{array}{llll}
q_{1}\left(K_{1} x(t)\right) & q_{2}\left(K_{2} x(t)\right) & \cdots & q_{m}\left(K_{m} x(t)\right)
\end{array}\right],
$$

where $q_{i}, i=1, \ldots, m$ are quantizers with different quantization density.

Without loss of generality, in this paper only a single-input case with $m=1$ is considered for simplicity, which can be generalized to a multi-input case. For a quantizer as given in the form of (2), as illustrated in [6], using the sector bound approach, the quantization error $e(t)$ can be characterized as

$$
e(t)=q(v(t))-v(t)=f(K x(t))-K x(t)=\Delta(t) K x(t),
$$

where $\Delta(t) \in[-\delta, \delta]$ with $\delta$ given by (4), so the closed-loop system with quantized feedback is given by

$$
x(t+1)=\left(A+A_{0} \xi(t)\right) x(t)+\left(B+B_{0} \xi(t)\right)(1+\Delta(t)) K x(t) .
$$

We mainly focus on the derivation of less conservative sufficient conditions for the system to achieve certain performance. To make the paper self-contained, the definitions for the system (8) to be mean-square stable and mean-square poly-quadratical stable are introduced.

Definition 1 The closed system (8) is called mean-square stable with quantized feedback control in the form of (6) if there exists a control Lyapunov function $V_{P}(x)=x^{T}(t) P x(t)$ satisfying

$$
E V_{P}(x(t+1))-E V_{P}(x(t))<0
$$

for all $x(t) \neq 0$ and the given quantization. 
Definition 2 The closed system (8) is called mean-square poly-quadratically stable with quantized control in the form of (6) if there exists a Lyapunov function

$$
V(x(t))=x^{T}(t)\left[\frac{\delta-\Delta(t)}{2 \delta} Q_{1}+\frac{\delta+\Delta(t)}{2 \delta} Q_{2}\right] x(t)=x^{T}(t) Q(t) x(t),
$$

where $Q_{1}$ and $Q_{2}$ are symmetric positive matrices with proper dimensions satisfying

$$
E V(x(t+1))-E V(x(t))<0
$$

for all $x(t) \neq 0$ and the given quantization.

Remark 1 When setting $Q_{1}=Q_{2}=P$, the control Lyapunov function proposed in Definition 2 reduces to the one given in Definition 1. We will show that the control Lyapunov function (10) can lead to less conservative conditions for the system (8) to be mean-square poly-quadratical stabilized than those deduced by the control Lyapunov function (10).

Problem formulation For the control Lyapunov function (10), deduce the conditions for the system (1) to be mean-square poly-quadratical stabilized via quantized feedback control in the form of (6).

\subsection{Stability analysis}

In this part, we give the conditions for the system (8) to achieve quantized mean-square poly-quadratical stability. First, a necessary and sufficient condition is deduced.

Theorem 1 For the discrete-time stochastic system (1) and the quantized state feedback control law in the form of (6), given a logarithmic quantizer as in (2), the closed-loop system (8) is mean-square poly-quadratically stable if and only if there exist matrices $Q_{1}>0, Q_{2}>$ $0, V_{1}$ and $V_{2}$ satisfying

$$
\begin{aligned}
& {\left[\begin{array}{ccc}
-Q_{1} & {[A+(1-\delta) B K]^{T} V_{i}} & \sigma\left[A_{0}+(1-\delta) B_{0} K\right]^{T} V_{i} \\
* & Q_{i}-V_{i}-V_{i}^{T} & 0 \\
* & 0 & Q_{i}-V_{i}-V_{i}^{T}
\end{array}\right]<0,} \\
& {\left[\begin{array}{ccc}
-Q_{2} & {[A+(1+\delta) B K]^{T} V_{i}} & \sigma\left[A_{0}+(1+\delta) B_{0} K\right]^{T} V_{i} \\
* & Q_{i}-V_{i}-V_{i}^{T} & 0 \\
* & 0 & Q_{i}-V_{i}-V_{i}^{T}
\end{array}\right]<0, \quad i \in\{1,2\} .}
\end{aligned}
$$

Proof According to Definition 2, for $E V(x(t))$ defined as in (10), the closed-loop system is mean-square poly-quadratically stable if

$$
E V(x(t+1))-E V(x(t))<0
$$

for all the $x(t) \neq 0$ and $\Delta(t) \in[-\delta, \delta]$. Plugging $E V(x(t))$ into (14), and by considering (6), we have

$$
\begin{aligned}
x^{T}(t) & {\left[[A+B K(1+\Delta(t))]^{T} Q(t+1)[A+B K(1+\Delta(t))]\right.} \\
& \left.+\sigma^{2}\left[A_{0}+B_{0} K(1+\Delta(t))\right]^{T} Q(t+1)\left[A_{0}+B_{0} K(1+\Delta(t))\right]-Q(t)\right] x(t)<0,
\end{aligned}
$$


which is equivalent to

$$
\begin{aligned}
& {[A+B K(1+\Delta(t))]^{T} Q(t+1)[A+B K(1+\Delta(t))]} \\
& \quad+\sigma^{2}\left[A_{0}+B_{0} K(1+\Delta(t))\right]^{T} Q(t+1)\left[A_{0}+B K_{0}(1+\Delta(t))\right]-Q(t)<0 .
\end{aligned}
$$

In the next part, we will show that the expressions in (10) and (16) hold if and only if (12) and (13) hold.

$(16) \Rightarrow(12)$ and (13): By the Schur complement, (16) is equivalent to

$$
\left[\begin{array}{ccc}
-Q(t) & {[A+(1+\Delta(t)) B K]^{T} Q(t+1)} & \sigma\left[A_{0}+(1+\Delta(t)) B_{0} K\right]^{T} Q(t+1) \\
* & -Q(t+1) & 0 \\
* & 0 & -Q(t+1)
\end{array}\right]<0 .
$$

Consider the following four cases:
(a) $\Delta(t)=-\delta, \quad \Delta(t+1)=-\delta$,
(b) $\quad \Delta(t)=-\delta, \quad \Delta(t+1)=\delta$,
(c) $\quad \Delta(t)=\delta, \quad \Delta(t+1)=-\delta$,
(d) $\quad \Delta(t)=\delta, \quad \Delta(t+1)=\delta$.

For cases (a) and (b), from (17) we have

$$
\left[\begin{array}{ccc}
-Q_{1} & {[A+(1-\delta) B K]^{T} Q_{i}} & \sigma\left[A_{0}+(1-\delta) B_{0} K\right]^{T} Q_{i} \\
* & -Q_{i} & 0 \\
* & 0 & -Q_{i}
\end{array}\right]<0, \quad i \in\{1,2\} .
$$

For cases (c) and (d), from (17) we have

$$
\left[\begin{array}{ccc}
-Q_{2} & {[A+(1+\delta) B K]^{T} Q_{i}} & \sigma\left[A_{0}+(1+\delta) B_{0} K\right]^{T} Q_{i} \\
* & -Q_{i} & 0 \\
* & 0 & -Q_{i}
\end{array}\right]<0, \quad i \in\{1,2\} .
$$

By selecting $V_{i}=V_{i}^{T}=Q_{i}$, we can obtain (12) and (13). Therefore, it can be concluded that if (16) holds, there must exist matrices $Q_{1}>0, Q_{2}>0, V_{1}$ and $V_{2}$ satisfying (12) and (13).

(12) and (13) $\Rightarrow(16)$ : Suppose there exist matrices $Q_{1}>0, Q_{2}>0, V_{1}$ and $V_{2}$ satisfying (12) and (13). First, as $Q_{i}>0$, we have $\left(V_{i}-Q_{i}\right)^{T} Q_{i}^{-1}\left(V_{i}-Q_{i}\right) \geq 0$, which implies

$$
-V_{i}^{T} Q_{i}^{-1} V_{i} \leq Q_{i}-V_{i}^{T}-V_{i}
$$

From (12) and (21) we have

$$
\begin{aligned}
& {\left[\begin{array}{ccc}
-Q_{1} & {[A+(1-\delta) B K]^{T} V_{i}} & \sigma\left[A_{0}+(1-\delta) B_{0} K\right]^{T} V_{i} \\
* & -V_{i}^{T} Q_{i}^{-1} V_{i} & 0 \\
* & 0 & -V_{i}^{T} Q_{i}^{-1} V_{i}
\end{array}\right]<0,} \\
& {\left[\begin{array}{ccc}
-Q_{2} & {[A+(1+\delta) B K]^{T} V_{i}} & \sigma\left[A_{0}+(1+\delta) B_{0} K\right]^{T} V_{i} \\
* & -V_{i}^{T} Q_{i}^{-1} V_{i} & 0 \\
* & 0 & -V_{i}^{T} Q_{i}^{-1} V_{i}
\end{array}\right]<0, \quad i \in\{1,2\} .}
\end{aligned}
$$


By multiplying $\operatorname{diag}\left\{I, Q_{i} V_{i}^{-1}, Q_{i} V_{i}^{-1}\right\}$ and $\operatorname{diag}\left\{I, V_{i}^{-1} Q_{i}, V_{i}^{-1} Q_{i}\right\}$ to the left- and right-hand side of (22) and (23), respectively, we get

$$
\begin{aligned}
& {\left[\begin{array}{ccc}
-Q_{1} & {[A+(1-\delta) B K]^{T} Q_{1}} & \sigma\left[A_{0}+(1-\delta) B_{0} K\right]^{T} Q_{1} \\
* & -Q_{1} & 0 \\
* & 0 & -Q_{1}
\end{array}\right]<0,} \\
& {\left[\begin{array}{ccc}
-Q_{1} & {[A+(1-\delta) B K]^{T} Q_{2}} & \sigma\left[A_{0}+(1-\delta) B_{0} K\right]^{T} Q_{2} \\
* & -Q_{2} & 0 \\
* & 0 & -Q_{2}
\end{array}\right]<0,} \\
& {\left[\begin{array}{ccc}
-Q_{2} & {[A+(1+\delta) B K]^{T} Q_{1}} & \sigma\left[A_{0}+(1+\delta) B_{0} K\right]^{T} Q_{1} \\
* & -Q_{1} & 0 \\
* & 0 & -Q_{1}
\end{array}\right]<0,} \\
& {\left[\begin{array}{ccc}
* Q_{2} & {[A+(1+\delta) B K]^{T} Q_{2}} & \sigma\left[A_{0}+(1+\delta) B_{0} K\right]^{T} Q_{2} \\
* & -Q_{2} & 0
\end{array}\right]<0 .}
\end{aligned}
$$

$(24) \times \frac{\delta-\Delta(t+1)}{2 \delta}$ and $(25) \times \frac{\delta+\Delta(t+1)}{2 \delta}$ we get

$$
\left[\begin{array}{ccc}
-Q_{1} & {[A+(1-\delta) B K]^{T} Q(\Delta(t+1))} & \sigma\left[A_{0}+(1-\delta) B_{0} K\right]^{T} Q(\Delta(t+1)) \\
* & -Q(\Delta(t+1)) & 0 \\
* & 0 & -Q(\Delta(t+1))
\end{array}\right]<0,
$$

$(26) \times \frac{\delta-\Delta(t+1)}{2 \delta}$ and $(27) \times \frac{\delta+\Delta(t+1)}{2 \delta}$ we get

$$
\left[\begin{array}{ccc}
-Q_{2} & {[A+(1+\delta) B K]^{T} Q(\Delta(t+1))} & \sigma\left[A_{0}+(1+\delta) B_{0} K\right]^{T} Q(\Delta(t+1)) \\
* & -Q(\Delta(t+1)) & 0 \\
* & 0 & -Q(\Delta(t+1))
\end{array}\right]<0 .
$$

$(28) \times \frac{\delta-\Delta(t)}{2 \delta}$ and $(29) \times \frac{\delta+\Delta(t)}{2 \delta}$ we can deduce that

$$
\left[\begin{array}{ccc}
-Q(\Delta(t)) & {[A+(1+\Delta(t)) B K]^{T} Q(\Delta(t+1))} & \sigma\left[A_{0}+(1+\Delta(t)) B_{0} K\right]^{T} Q(\Delta(t+1)) \\
* & -Q(\Delta(t+1)) & 0 \\
* & 0 & -Q(\Delta(t+1))
\end{array}\right]
$$

$<0$.

The proof is completed.

\subsection{Controller design}

In the above section, the controller is assumed to be known for the stability analysis. In practical situations, however, the controller has to be designed to guarantee the closedloop system to achieve stability. The following theorem provides a controller design method based on Theorem 1 .

Theorem 2 Consider the system (1) and the state feedback control law in (5). Given a logarithmic quantizer as in (2), the closed-loop system (6) is mean-square poly-quadratically 
stabilized if there exist matrices $\bar{Q}_{1}>0, \bar{Q}_{2}>0, V$ and $K$ satisfying

$$
\begin{aligned}
& {\left[\begin{array}{ccc}
-\bar{Q}_{1} & {[A V+(1-\delta) B \bar{K}]^{T}} & \sigma\left[A_{0} V+(1-\delta) B_{0} \bar{K}\right]^{T} \\
* & \bar{Q}_{i}-V-V^{T} & 0 \\
* & 0 & \bar{Q}_{i}-V-V^{T}
\end{array}\right]<0,} \\
& {\left[\begin{array}{ccc}
-\bar{Q}_{2} & {[A V+(1+\delta) B \bar{K}]^{T}} & \sigma\left[A_{0} V+(1+\delta) B_{0} \bar{K}\right]^{T} \\
* & \bar{Q}_{i}-V-V^{T} & 0 \\
* & 0 & \bar{Q}_{i}-V-V^{T}
\end{array}\right]<0, \quad i \in\{1,2\} .}
\end{aligned}
$$

In this situation, the controller can be designed as

$$
K=\bar{K} V^{-1} \text {. }
$$

Proof Suppose that there exist matrices $\bar{Q}_{1}>0$ and $\bar{Q}_{2}>0, V$ and $\bar{K}$ satisfying (31) and (32). From the $(2,2)$ block, we know that $\bar{Q}_{i}-V-V^{T}<0$, which means $V+V^{T}>\bar{Q}_{i}>0$, so $V$ is nonsingular. Performing $\operatorname{diag}\left\{V^{-T}, V^{-T}, V^{-T}\right\}$ and $\operatorname{diag}\left\{V^{-1}, V^{-1}, V^{-1}\right\}$ to (31) and (32), respectively, yields

$$
\begin{aligned}
& {\left[\begin{array}{ccc}
-V^{-T} \bar{Q}_{1} V^{-1} & V^{-T}[A V+(1-\delta) B \bar{K}]^{T} V^{-1} & \sigma V^{-T}\left[A_{0} V+(1-\delta) B_{0} \bar{K}\right]^{T} V^{-1} \\
* & V^{-T} \bar{Q}_{i} V^{-1}-V^{-T}-V^{-1} & 0 \\
* & 0 & V^{-T} \bar{Q}_{i} V^{-1}-V^{-T}-V^{-1}
\end{array}\right]} \\
& <0, \\
& {\left[\begin{array}{ccc}
-V^{-T} \bar{Q}_{2} V^{-1} & V^{-T}[A V+(1+\delta) B \bar{K}]^{T} V^{-1} & \sigma V^{-T}\left[A_{0} V+(1+\delta) B_{0} \bar{K}\right]^{T} V^{-1} \\
* & V^{-T} \bar{Q}_{i} V^{-1}-V^{-T}-V^{-1} & 0 \\
* & 0 & V^{-T} \bar{Q}_{i} V^{-1}-V^{-1}-V^{T}
\end{array}\right]} \\
& <0, \quad i \in\{1,2\} .
\end{aligned}
$$

By defining the following matrix variables: $Q_{i}=V^{-T} \bar{Q}_{i} V^{-1}, V_{i}=V^{-1}, K=\bar{K} V^{-1}$, if there exist matrices $Q_{1}>0, Q_{2}>0, V_{1}$ and $V_{2}$ satisfying (12) and (13), and using the controller gain given in (33), the system (8) can achieve mean-square poly-quadratically stability.

Theorem 2 is based on Theorem 1 by setting $V_{1}=V_{2}=V$, which increases the conservativeness; the following theorem gives a less conservative condition.

Theorem 3 Consider the system in (1) and the state feedback control law in (5). Given a logarithmic quantizer as in (2), the closed-loop system in (8) is mean-square polyquadratically stable if there exist matrices $Q_{i}>0, X_{i}>0, \bar{V}_{i}$ and $K$ satisfying

$$
\left[\begin{array}{ccccc}
-Q_{1} & {[A+(1-\delta) B K]^{T}} & 0 & {\left[A_{0}+(1-\delta) B_{0} K\right]^{T}} & 0 \\
* & -\bar{V}_{i}^{T}-\bar{V}_{i} & \bar{V}_{i}^{T} & 0 & 0 \\
* & * & -X_{i} & 0 & 0 \\
* & * & * & -\bar{V}_{i}^{T}-\bar{V}_{i} & \bar{V}_{i}^{T} \\
* & * & * & * & -X_{i}
\end{array}\right]<0,
$$




$$
\begin{aligned}
& \left.\begin{array}{ccccc}
-Q_{2} & {[A+(1-\delta) B K]^{T}} & 0 & {\left[A_{0}+(1-\delta) B_{0} K\right]^{T}} & 0 \\
* & -\bar{V}_{i}^{T}-\bar{V}_{i} & \bar{V}_{i}^{T} & 0 & 0 \\
* & * & -X_{i} & 0 & 0 \\
* & * & * & -\bar{V}_{i}^{T}-\bar{V}_{i} & \bar{V}_{i}^{T} \\
* & * & * & * & -X_{i}
\end{array}\right]<0, \quad i \in\{1,2\}, \\
& X_{i} Q_{i}=I .
\end{aligned}
$$

Proof First, from the $(2,2)$ block of (36), we can know that $\bar{V}_{i}$ is nonsingular. By multiplying $\operatorname{diag}\left\{I, \bar{V}_{i}^{-T}, I, \bar{V}_{i}^{-T}, I\right\}$ and $\operatorname{diag}\left\{I, \bar{V}_{i}^{-1}, I, \bar{V}_{i}^{-1}, I\right\}$ to the left- and right-hand side of (36) and (37), with the Schur complement and the constraint (38), and defining $\bar{V}_{i}^{-1}=V_{i}$, we can get the theorem.

Remark 2 It is worth noting that when $\delta_{\max }$ is known, the conditions in Theorem 1 are linear matrix inequalities over the matrix variables $Q_{1}>0, Q_{2}>0, V_{1}$ and $V_{2}$. When Theorem 1 is used to compute the coarsest quantization density $\delta_{\max }$ such that the closed-loop quantized system is mean-square poly-quadratically stable, that is, (12) and (13) are bilinear matrix inequalities. In this case, a line search (such as the bisection method) has to be performed to the variables $\delta$ in (12) and (13), and find $\delta_{\max }$ iteratively, which can be referred to [13-16].

\subsection{Illustrative example}

In this part, an example is given to show that the new proposed Lyapunov function can lead to less conservative conditions of the quantization density for the system to achieve stability.

Example 1 For the stochastic discrete-time system (1), consider the scalar case of the following form:

$$
\begin{aligned}
& A=A_{0}=\left[\begin{array}{cccc}
0.8 & -0.25 & 0 & 1 \\
1 & 0 & 0 & 0 \\
-0.8 & 0.5 & 0.2 & -1.03 \\
0 & 0 & 1 & 0
\end{array}\right], \\
& B=B_{0}=\left[\begin{array}{llll}
0 & 0 & 1 & 0
\end{array}\right]^{T}, \\
& E \xi(t)=0, \quad E \xi^{2}(t)=\sigma^{2}=0.04 .
\end{aligned}
$$

It can be proved that the system without control part is unstable in the mean-square sense. Suppose that the state-feedback in (5) is given by $K=[0.8-0.501]$, and the quantizer we use is logarithmic in the form of (2). We want to determine the maximum sector bound $\delta_{\max }$ below which the stochastic system with quantized state feedback is mean-square asymptotically stable. Table 1 gives the maximum bound of $\delta_{\max }$ using the Lyapunov function related to the quantization density proposed in this paper and the general control Lyapunov function. 
Table 1 Comparison of quantization density

\begin{tabular}{lll}
\hline Methods & $\boldsymbol{\delta}_{\mathbf{m a x}}$ & $\boldsymbol{\rho}_{\text {inf }}$ \\
\hline Quadratic approach & 0.4450 & 0.3841 \\
Quantization dependent approach & 0.4996 & 0.3337 \\
\hline
\end{tabular}

\section{Extension to $H_{\infty}$ performance analysis}

For the system

$$
\begin{aligned}
& x(t+1)=\left(A+A_{0} \xi(t)\right) x(t)+\left(B+B_{0} \xi(t)\right) u(t)+G w(t), \\
& z(t)=C x(t)+D u(t)+F w(t),
\end{aligned}
$$

where the state $x(t)$, the input $u(t)$ and the system noise $\xi(t)$ are defined as those of the system (1), $z(t) \in R^{n}$ is the control output. $A, A_{0}, B, B_{0}, C, D, G, F$ are system matrices with proper dimensions. Suppose the quantizer is given to be logarithmic in the form of (24) and the quantization density is known, so the closed-loop system with the quantized state feedback control is given as follows:

$$
\begin{aligned}
& x(t+1)=\left(A+A_{0} \xi(t)\right) x(t)+\left(B+B_{0} \xi(t)\right)(1+\Delta(t)) K x(t)+G w(t), \\
& z(t)=C x(t)+D(1+\Delta(t)) K x(t)+F w(t),
\end{aligned}
$$

where $\Delta(t) \in[-\delta, \delta]$. Defining $W=\{w(t)\} \in l_{2}[0, \infty)$, the objective of this part is to derive the conditions for the system (42) and (43) to be mean-square asymptotically stable with an $H_{\infty}$ disturbance attention level $\gamma$, that is, $\|z(t)\|_{2}<\gamma\|w(t)\|_{2}$ for all the nonzero $w(t) \in$ $l_{2}[0, \infty)$ and for all the $\Delta(t) \in[-\delta, \delta]$ under zero conditions.

Theorem 4 For the system (40) and (41), considering the control law as given in (5), given a logarithmic quantizer as in (2), the closed-loop system in (42) and (43) is mean-square stable with an $H_{\infty}$ disturbance attention level $\gamma$ if there exist matrices $Q_{1}=Q_{1}^{T}>0, Q_{2}=$ $Q_{2}^{T}>0, V_{1}$ and $V_{2}$ satisfying

$$
\begin{aligned}
& {\left[\begin{array}{ccccc}
-Q_{1} & 0 & {[A+(1-\delta) B K]^{T} V_{i}} & {[C+(1-\delta) D K]^{T}} & \sigma\left[A_{0}+(1-\delta) B_{0} K\right]^{T} V_{i} \\
* & -\gamma^{2} I & G^{T} V_{i} & F^{T} & 0 \\
* & 0 & Q_{i}-V_{i}-V_{i}^{T} & 0 & 0 \\
* & * & * & -I & 0 \\
* & * & * & * & Q_{i}-V_{i}-V_{i}^{T}
\end{array}\right]} \\
& <0 \text {, } \\
& {\left[\begin{array}{ccccc}
-Q_{2} & 0 & {[A+(1+\delta) B K]^{T} V_{i}} & {[C+(1+\delta) D K]^{T}} & \sigma\left[A_{0}+(1+\delta) B_{0} K\right]^{T} V_{i} \\
* & -\gamma^{2} I & G^{T} V_{i} & F^{T} & 0 \\
* & 0 & Q_{i}-V_{i}-V_{i}^{T} & 0 & 0 \\
* & * & * & -I & 0 \\
* & * & * & * & Q_{i}-V_{i}-V_{i}^{T}
\end{array}\right]} \\
& <0, \quad i \in\{1,2\} \text {. }
\end{aligned}
$$

Proof The theorem is proven based on the Lyapunov function defined in (10). First, (44) and (45) imply (12) and (13), which guarantees the closed-loop system in (42) and (43) to 
be mean-square stable by Theorem 1 . To prove the $H_{\infty}$ performance, assume zero initial conditions and consider the following index:

$$
\begin{aligned}
\aleph & =\sum_{0}^{\infty}\left[E z^{T}(t) z(t)-\gamma^{2} E w^{T}(t) w(t)\right] \\
& \leq \sum_{0}^{\infty}\left[E z^{T}(t) z(t)-\gamma^{2} E w^{T}(t) w(t)+\nabla E V(x(t))\right],
\end{aligned}
$$

where

$$
\nabla E V(x(t))=E x^{T}(t+1) Q(\Delta(t+1)) x(t+1)-x^{T}(t) Q(\Delta(t)) x(t) .
$$

Then, along the solutions of (42) and (43), we have

$$
\aleph=\sum_{0}^{\infty} \eta^{T}(t) \Pi \eta(t)
$$

with $\eta(t)=\left[\begin{array}{c}x(t) \\ w(t)\end{array}\right], \Pi=\left(\begin{array}{cc}\Pi_{11} & \Pi_{12} \\ * & \Pi_{22}\end{array}\right)$, where

$$
\begin{aligned}
\Pi_{11}= & (A+(1+\Delta(t)) B K)^{T} Q(\Delta(t+1))(A+(1+\Delta(t)) B K)-Q(\Delta(t)) \\
& +\sigma^{2}\left(A_{0}+(1+\Delta(t)) B_{0} K\right)^{T} Q(\Delta(t+1))\left(A_{0}+(1+\Delta(t)) B_{0} K\right) \\
& +(C+(1+\Delta(t)) D K)^{T}(C+(1+\Delta(t)) D K), \\
\Pi_{12}= & (A+(1+\Delta(t)) B K)^{T} Q(\Delta(t+1)) G+(C+(1+\Delta(t)) D K)^{T} F, \\
\Pi_{22}= & G^{T} Q(\Delta(t+1)) G+F^{T} F-\gamma^{2} I .
\end{aligned}
$$

On the other hand, by similar reasoning as in the proof of Theorem 1, we can conclude from (42) and (43) that $\Pi<0$. Then from (48) we know that $\aleph<0$ for all nonzero $w(t) \in$ $l_{2}[0, \infty)$. The proof is completed.

\section{Conclusion}

The problem of quantized state-feedback control for a stochastic system with multiplicative noises has been investigated through a quantization-dependent approach. Conditions for mean-square poly-quadratical stability are obtained by introducing a new quantization-dependent Lyapunov function approach for linear state feedback with a logarithmic quantizer, which are shown to be less conservative than those derived by a common Lyapunov function. Moreover, $H_{\infty}$ performance analysis has also been proposed in the quantization-dependent framework. However, it is worth pointing out that though less conservative conditions are obtained, different from the derivation of the coarsest quantizer, the explicit relation of the system matrices and quantization density is not given. The analysis of relation between the quantization density and the system matrices and the statistical properties of noises in the proposed quantization-dependent framework is a subject worth further researching. 


\section{Competing interests}

The authors declare that they have no competing interests.

\section{Authors' contributions}

LW carried out the proof of the main part of this article, YY corrected the manuscript and participated in its design and coordination. All authors have read and approved the final manuscript.

\section{Acknowledgements}

We would like to thank the editor-in-chief, the associate editor and the reviewers for their valuable comments on the paper which have led to significant improvement on the presentation and quality of the paper. This work is supported by the Taishan Scholar Construction Engineering by Shandong Government, the National Natural Science Foundation (No. 61174141), and the Major State Basic Research Development Program of China (973 Program) (No. 2009cb320600), Yangtse Rive Scholar Bonus Schemes (No. 31400080963017), National Natural Science Foundation (No. 61034007).

Received: 20 July 2012 Accepted: 18 December 2012 Published: 23 January 2013

\section{References}

1. Kalman, RE: Nonlinear aspects of sampled-data control systems. In: Proc. Symposium on Nonlinear Circuit Theory VII. Polytechnic Press, New York (1956)

2. Lewis, JB, Tou, JT: Optimum sampled-data systems with quantized control signals. IEEE Trans. Appl. Ind. 82(67), 229-233 (1963)

3. Elia, N, Mitter, S: Stabilization of linear systems with limited information. IEEE Trans. Autom. Control 46(9), 1384-1400 (2001)

4. Tsumura, $\mathrm{K}$, Ishii, $\mathrm{H}$, Hoshina, $\mathrm{H}$ : Tradeoffs between quantization and packet loss in networked control of linear systems. Automatica 45, 2963-2970 (2009)

5. Wei, L, Fu, M, Zhang, H: Quantized stabilization for stochastic discrete-time systems with multiplicative noises. Int. J. Robust Nonlinear Control (2012). doi:10.1002/rnc.2778

6. Fu, M, Xie, L: The sector bound approach to quantized feedback control. IEEE Trans. Autom. Control 50(11), 1698-1711 (2005)

7. Hayakawa, T, Ishii, H, Tsumura, K: Adaptive quantized control for linear uncertain discrete-time systems. Automatica 45, 692-700 (2009)

8. Hayakawa, T, Ishii, H, Tsumura, K: Adaptive quantized control for nonlinear uncertain systems. Syst. Control Lett. 58, 625-632 (2009)

9. Gao, H, Chen, T: A new approach to quantized feedback control systems. Automatica 44, 534-542 (2008)

10. Zhou, B, Duan, G, Lam, J: On the absolute stability approach to quantized feedback control. Automatica 46, 337-346 (2010)

11. You, K, Su, W, Fu, M, Xie, L: Attainability of the minimum data rate for stabilization of linear systems via logarithmic quantization. Automatica 47(1), 170-176 (2011)

12. Niu, Y, Jia, T, Wang, X, Yang, F: Output-feedback control design for NCSs subject to quantization and dropout. Inf. Sci. 179(21), 3804-3813 (2009)

13. Feng, G: Controller design and analysis of uncertain piecewise-linear systems. IEEE Trans. Circuits Syst. I 49(2), 224-232 (2002)

14. Kocvara, M, Stingl, M: Pennon: a code for convex nonlinear and semidefinite programming. Optim. Methods Softw. 18(3), 317-333 (2003)

15. Zhu, Y, Li, DQ, Feng, G: $H_{\infty}$ controller synthesis of uncertain piecewise continuous-time linear systems. IEE Proc., Control Theory Appl. 152(5), 513-519 (2005)

16. Zhang, H, Feng, G, Dang, C: Stability analysis and $H_{\infty}$ control for uncertain stochastic piecewise-linear systems. IET Control Theory Appl. 3(8), 1059-1069 (2009)

doi:10.1186/1687-1847-2013-20

Cite this article as: Wei and Yang: A new approach to quantized stabilization of a stochastic system with multiplicative noise. Advances in Difference Equations 2013 2013:20.

\section{Submit your manuscript to a SpringerOpen ${ }^{\circ}$ journal and benefit from:}

- Convenient online submission

- Rigorous peer review

- Immediate publication on acceptance

- Open access: articles freely available online

- High visibility within the field

- Retaining the copyright to your article 